\title{
Mutation causing severe myasthenia reveals functional asymmetry of AChR signature cystine loops in agonist binding and gating
}

\author{
Xin-Ming Shen, ${ }^{1}$ Kinji Ohno, ${ }^{1}$ Akira Tsujino, ${ }^{1}$ Joan M. Brengman, ${ }^{1}$ Monique Gingold, ${ }^{2}$ \\ Steven M. Sine, ${ }^{3}$ and Andrew G. Engel ${ }^{1}$ \\ ${ }^{1}$ Department of Neurology and Neuromuscular Research Laboratory, Mayo Clinic, Rochester, Minnesota, USA \\ ${ }^{2}$ Childrens Neurology Services, Morgantown, West Virginia, USA \\ ${ }^{3}$ Receptor Biology Laboratory, Department of Physiology and Biophysics, Mayo Clinic, Rochester, Minnesota, USA
}

We describe a highly disabling congenital myasthenic syndrome (CMS) associated with rapidly decaying, low-amplitude synaptic currents, and trace its cause to a valine to leucine mutation in the signature cystine loop (cys-loop) of the AChR $\alpha$ subunit. The recently solved crystal structure of an ACh-binding protein places the cys-loop at the junction between the extracellular ligand-binding and transmembrane domains where it may couple agonist binding to channel gating. We therefore analyzed the kinetics of ACh-induced single-channel currents to identify elementary steps in the receptor activation mechanism altered by the $\alpha \mathrm{V} 132 \mathrm{~L}$ mutation. The analysis reveals that $\alpha \mathrm{V} 132 \mathrm{~L}$ markedly impairs ACh binding to receptors in the resting closed state, decreasing binding affinity for the second binding step 30-fold, but attenuates gating efficiency only about twofold. By contrast, mutation of the equivalent valine residue in the $\delta$ subunit impairs channel gating approximately fourfold with little effect on ACh binding, while corresponding mutations in the $\beta$ and $\varepsilon$ subunits are without effect. The unique functional contribution of the $\alpha$ subunit cys-loop likely owes to its direct connection via a $\beta$ strand to $\alpha \mathrm{W} 149$ at the center of the ligand-binding domain. The overall findings reveal functional asymmetry between cys-loops of the different AChR subunits in contributing to ACh binding and channel gating.

J. Clin. Invest. 111:497-505 (2003). doi:10.1172/JCI200316997.

\section{Introduction}

Congenital myasthenic syndromes (CMSs) are heterogeneous disorders caused by presynaptic, synaptic, or postsynaptic defects (1). Postsynaptic CMSs can be broadly classified according to whether the response to $\mathrm{ACh}$ is increased or decreased relative to control responses. An increased response is observed in slowchannel syndromes due to prolonged activation episodes of AChR. A decreased response is observed with AChR deficiency due to mutations in AChR subunit genes (2) or in rapsyn (3), and in the fast-channel syndromes. Fast-channel syndromes are characterized by attenuated and abnormally rapidly decaying endplate (EP) currents, abnormally brief single-channel currents, and decreased probability of channel opening.

Received for publication September 26, 2002, and accepted in revised form November 26, 2002.

Address correspondence to: Andrew G. Engel, Department of Neurology, Mayo Clinic, Rochester, Minnesota 55905, USA. Phone: (507) 284-5102; Fax: (507) 284-5831;

E-mail: age@mayo.edu.

Conflict of interest: The authors have declared that no conflict of interest exists.

Nonstandard abbreviations used: cystine loop (cys-loop); congenital myasthenic syndrome (CMS); endplate (EP); acetylcholinesterase (AChE); $\alpha$-bungarotoxin ( $\alpha$-bgt); endplate potential (EPP); endplate current (EPC); miniature endplate potential (MEPP); miniature endplate current (MEPC); gating equilibrium constant $(\theta)$; open probability $\left(P_{\text {open }}\right)$.
Several molecular mechanisms underlying fastchannel syndromes have been identified. At the ligand-binding site formed by $\alpha$ and $\varepsilon$ subunits, the mutation $\varepsilon P 121 \mathrm{~L}$ reduces ACh affinity for the open channel state and slows the rate of channel opening, resulting in a moderately severe myasthenic phenotype (4). Also at the $\alpha-\varepsilon$ site, the mutation $\varepsilon D 175 \mathrm{~N}$ reduces ACh affinity of the resting closed state and impairs gating efficiency, while the nearby mutation $\varepsilon N 182 Y$ increases ACh affinity for the resting closed state and also impairs gating (5). The phenotypic consequences of these combined mutations are also moderately severe. At the ligand-binding site formed by the $\alpha$ and $\delta$ subunits, the mutation $\delta$ E59K likely reduces ACh affinity (6), but the altered steps in the activation process have not been identified. This mutation was shown to result in hypomotility in utero, multiple congenital joint contractures, and neonatal respiratory distress, but the patient subsequently improved and could walk short distances. In the third transmembrane domain of the $\alpha$ subunit, the mutation $\alpha \mathrm{V} 285 \mathrm{I}$ impairs gating efficiency (7) but results in a mild phenotype. Finally, in the amphipathic helix of the long cytoplasmic loop of the $\varepsilon$ subunit, either a duplication of codons 413-418 (8) or an $\alpha \mathrm{A} 411 \mathrm{P}$ missense mutation (9) result in heterogeneous channel gating kinetics; the phenotypic consequences are mild to moderately severe. 
Here we describe a fast-channel CMS caused by an $\alpha V 132 \mathrm{~L}$ mutation in the signature cystine loop (cysloop) of the AChR $\alpha$ subunit that is more severely disabling than any previously reported fast-channel CMS. The cys-loop, formed by a disulfide bond between cysteines 128 and 142 of the $\alpha$ subunit and equivalent cysteines in non- $\alpha$ subunits, is one of the most highly conserved structural domains among members of the AChR superfamily (10). Previous studies suggested that the cys-loop is required for assembly of AChR subunits and formation of the ligand-binding sites (11-13), but $\alpha$ or $\beta$ subunits lacking the cys-loop disulfide bond can still form stable complexes with other subunits $(14,15)$. Other reports implicate the cys-loop in $\alpha$-neurotoxin binding $(11,15,16)$, but the cys-loop is not fully exposed on the surface of the native receptor (17), and recent atomic structural evidence places it remote from the toxin-binding site (18). The present study uses single-channel kinetic analysis to identify elementary functional steps altered by $\alpha V 132 \mathrm{~L}$, and show that the mutation profoundly impairs ACh binding to the resting closed state but only slightly attenuates channel gating. Mutations of equivalent residues in $\beta$ and $\varepsilon$ subunits are without effect, but mutation of the $\delta$ subunit markedly impairs channel gating. The overall findings reveal distinct contributions of cys-loops of the different $\mathrm{AChR}$ subunits to ACh binding and channel gating.

\section{Methods}

Muscle specimens. Intercostal muscle specimens were obtained intact from origin to insertion from the patient and from control subjects without muscle disease undergoing thoracic surgery. All human studies were in accord with the guidelines of the Institutional Review Board of the Mayo Clinic.

$\mathrm{AChR}$ and acetylcholinesterase (AChE) were detected in cryostat sections by two-color fluorescence (19). EPs were localized for electron microscopy and analyzed by established methods $(20,21)$. Peroxidaselabeled $\alpha$-bungarotoxin $(\alpha$-bgt) was used for the ultrastructural localization of AChR (22). The number of AChR's per EP was measured with [125I] $\alpha$-bgt (23).

Electrophysiology of muscle specimens. Recordings of EP potentials (EPPs) and EP currents (EPCs) and estimates of the number of transmitter quanta released by nerve impulse were performed as described elsewhere $(23,24)$, except that the amplitude of the miniature EPPs (MEPPs) and currents (MEPCs) were estimated from the quantal components of the EPP and EPC, respectively (25). Single-channel patchclamp recordings from the EP were performed in the cell-attached mode as described (26).

Mutation analysis. We directly sequenced the $\mathrm{AChR} \alpha$ and $\varepsilon$ subunit genes using genomic DNA (27). $\alpha V 132 \mathrm{~L}$ results in gain of an $M n l \mathrm{I}$ site, and $\alpha 381 \mathrm{delC}$ results in loss of a Bst4CI site. Therefore we screened for these mutations in relatives by restriction analysis. We also screened for $\alpha$ V132L in 200 normal alleles using allele-specific PCR.
Construction and expression of wild-type and mutant $A C h R$ 's. Sources of human $\alpha, \beta, \delta$, and $\varepsilon$ subunit cDNAs were as previously described $(28,29)$. All four cDNAs were subcloned into the CMV-based expression vector pRBG4 (4) for expression in human embryonic kidney fibroblast (HEK 293) cells. The $\alpha$ V132L, $\alpha 381$ delC, $\alpha$ V132I, $\beta$ V132L, $\delta$ V134L, and $\varepsilon V 132 \mathrm{~L}$ mutations were engineered into the wild-type $\alpha, \beta, \delta$, and $\varepsilon$ subunit cDNA in pRBG4 using the QuikChange Site-Directed Mutagenesis Kit (Stratagene, La Jolla, California, USA) (30). Presence of the desired mutation and absence of unwanted mutations were confirmed by sequencing the entire inserts. HEK cells were transfected with mutant and complementary wild-type AChR subunit cDNAs using calcium phosphate precipitation (31).
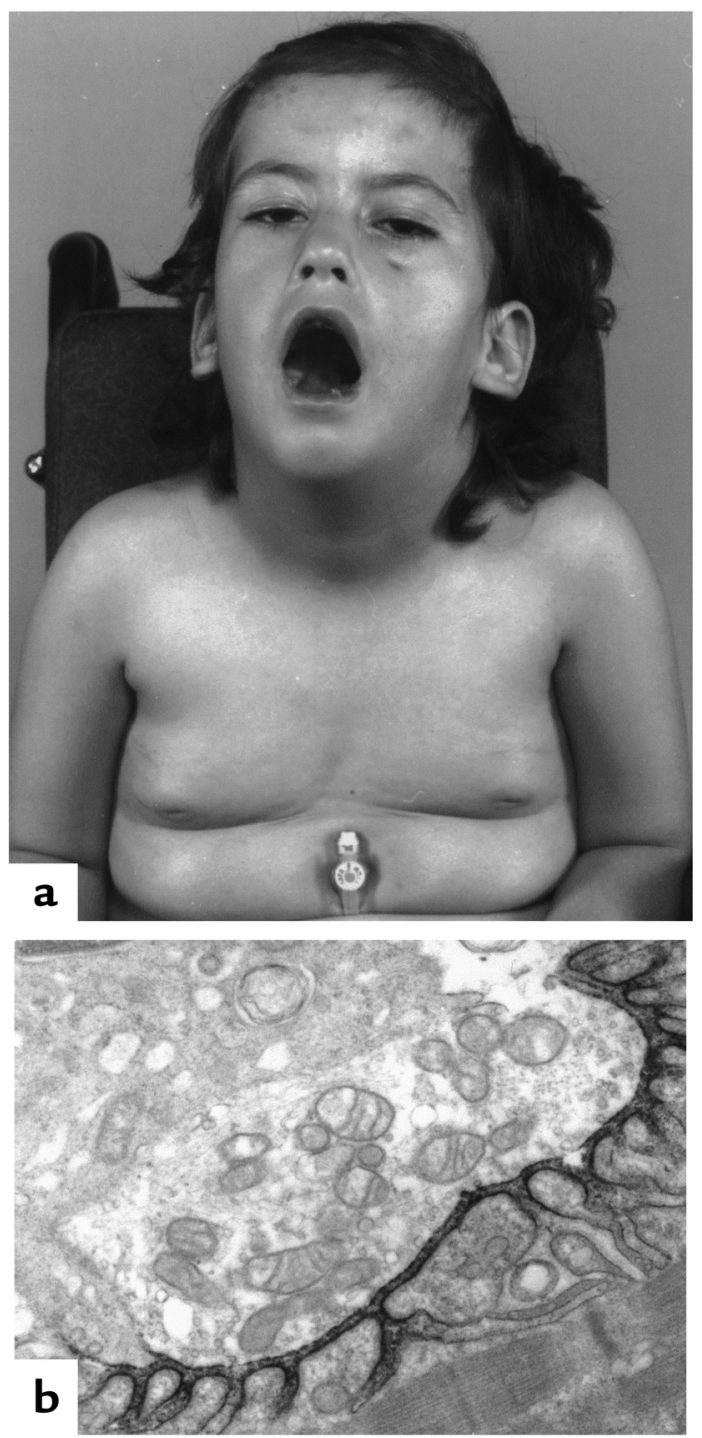

\section{Figure 1}

(a) Patient at age 4. Note eyelid ptosis, facial diplegia, open mouth, gothic palate, and gastrostomy. (b) Ultrastructural localization of EP AChR with peroxidase-labeled $\alpha$-bgt. The density and distribution of AChR on the junctional folds is normal. $\times 9,500$. 
Table 1

EP studies

\begin{tabular}{|c|c|c|}
\hline & Controls & Patient \\
\hline $\begin{array}{l}{\left[{ }^{125} \mid\right] \alpha \text {-bgt }} \\
\text { binding sites/EP }\end{array}$ & $12.82 \pm 0.79 \mathrm{E} 6(13)$ & $10.7 \mathrm{E} 6$ \\
\hline AChR index & $3.3 \pm 0.08(155)$ & $3.20 \pm 0.19(50)$ \\
\hline$m^{\mathrm{A}}$ & $31 \pm 1(190)^{B}$ & $26 \pm 2.10(26)$ \\
\hline MEPP amplitude $(\mathrm{mV})^{\mathrm{B}}$ & $1.00 \pm 0.025(165)$ & $0.096 \pm 0.011(26$ \\
\hline MEPC amplitude $(n A)^{C}$ & $3.95 \pm 0.10(79)$ & $0.32 \pm 0.044$ \\
\hline \multicolumn{3}{|l|}{ Bursts ${ }^{D}$} \\
\hline$\tau_{1}(\mathrm{~ms})$ & $0.12 \pm 0.012(32)$ & $0.44 \pm 0.057(3)$ \\
\hline Area & $0.16 \pm 0.014$ & $0.86 \pm 0.059$ \\
\hline$\tau_{2}(\mathrm{~ms})$ & $3.04 \pm 0.175(34)$ & $1.35 \pm 0.32(2)$ \\
\hline Area & $0.85 \pm 0.015$ & $0.14 \pm 0.059$ \\
\hline
\end{tabular}

Values indicate mean \pm SEM. Measurements were taken at $29^{\circ} \mathrm{C} \pm 0.5^{\circ} \mathrm{C}$ for EPPs and at $22^{\circ} \mathrm{C} \pm 0.5^{\circ} \mathrm{C}$ for EPCs and single-channel recordings. Numbers in parentheses indicate number of patients studied for [ $\left.{ }^{125} \mid\right] \alpha$-bgt binding sites/EP, number of EP regions used for AChR index, and number of EPs included in other measurements. ${ }^{\mathrm{A}} \mathrm{Quantal}$ content of EPP at $1 \mathrm{~Hz}$ stimulation corrected for a resting membrane potential of $-80 \mathrm{mV}$, nonlinear summation, and non-Poisson release. ${ }^{B}$ Corrected for resting membrane potential of -80 $\mathrm{mV}$; MEPP amplitude in patient was determined from quantal component of EPP and corrected for a fiber diameter of $50 \mu \mathrm{m}$. ${ }^{C} \mathrm{MEPC}$ amplitude in patient was estimated from quantal component of EPC. ${ }^{\mathrm{D} A C h}, 1 \mu \mathrm{M}$; pipette potential, $80 \mathrm{mV}$; bandwidth, $5.8 \mathrm{kHz}$. The $\tau_{1}$ component of bursts was absent at two control EPs and the $\tau_{2}$ component was absent at one patient EP. Values in bold indicate dominant components of bursts.

Measurements of $\alpha$-bgt binding. The total number of $\left.{ }^{[25} \mathrm{I}\right] \alpha$-bgt sites on the surface of transfected HEK cells and ACh competition against the initial rate of $\left.{ }^{125} \mathrm{I}\right] \alpha$-bgt binding were determined as previously described (4). ACh competition measurements were analyzed according to the Hill equation: $1-Y=1 /(1+$ $\left.\left([\mathrm{ACh}] / K_{\mathrm{ov}}\right)^{n}\right)$, where $Y$ is fractional occupancy by ACh, $n$ is the Hill coefficient, and $K_{\mathrm{ov}}$ is an overall dissociation constant.

Patch-clamp recordings from AChR's expressed in HEK cells. Recordings were obtained in the cell-attached configuration at a membrane potential of $-80 \mathrm{mV}$ at $22^{\circ} \mathrm{C}$, with bath and pipette solutions containing $142 \mathrm{mM} \mathrm{KCl}, 5.4 \mathrm{mM} \mathrm{NaCl}, 1.8 \mathrm{mM} \mathrm{CaCl}_{2}, 1.7 \mathrm{mM}$ $\mathrm{MgCl}_{2}$, and $10 \mathrm{mM}$ HEPES, pH 7.4 (4, 31). Singlechannel currents were recorded using an Axopatch 200A amplifier (Axon Instruments Inc., Union City, California, USA) at a bandwidth of $50 \mathrm{kHz}$, digitized at $5-\mu$ s intervals using a Digidata 1200A (Axon Instruments Inc.), and recorded to hard disk using the program pCLAMP (Axon Instruments Inc.). Records were analyzed at a uniform bandwidth of $11.7 \mathrm{kHz}$ with TAC $\times 4.0 .9$ software (Bruxton Corporation, Seattle, Washington, USA).

To estimate rate constants underlying AChR activation (see Results), we used desensitizing concentrations of ACh that cause events from a single channel to cluster into identifiable activation episodes (32). Clusters were identified as series of closely spaced openings preceded and followed by closed intervals greater than a specified critical time. The latter was obtained from the intersection of the predominant closed time component with the succeeding closed time component in the histogram. Clusters with fewer than five openings were excluded from analysis. Individual clusters were examined for homogeneity by determining the mean open probability and open duration for each cluster, and clusters within two standard deviations of the mean were accepted for further analysis $(33,34)$. The resulting open and closed intervals for a given set of clusters were analyzed according to kinetic schemes of receptor activation using the program MIL, which uses an interval-based maximum likelihood method that also corrects for missed events (33). A dead time of $23 \mu \mathrm{s}$ was superimposed on all recordings. For each type of AChR, single-channel dwell times obtained at several concentrations of ACh were fitted simultaneously. Data for different AChR's were obtained at the following concentrations of ACh (in $\mu \mathrm{M}$ ): wild type, $\alpha \mathrm{V} 132 \mathrm{I}, \delta \mathrm{V} 134 \mathrm{~L}, \beta \mathrm{V} 132 \mathrm{~L}$, and EV132L at 10, 20, 30, 50, 70, 100, 200, and 300; and $\alpha \mathrm{V} 132 \mathrm{~L}$ at $50,70,100,200,300$, and 1,000 . Individual recordings contained 4,913-20,651 events in 57-311 clusters. To check the final set of fitted rate constants, probability density curves were calculated from fitted rate constants and superimposed on the experimental dwell-time histograms.

\section{Results}

Characteristics of CMS patient. A 4-year-old girl has had life-threatening myasthenic symptoms since birth, requiring frequent ventilator support. She cannot

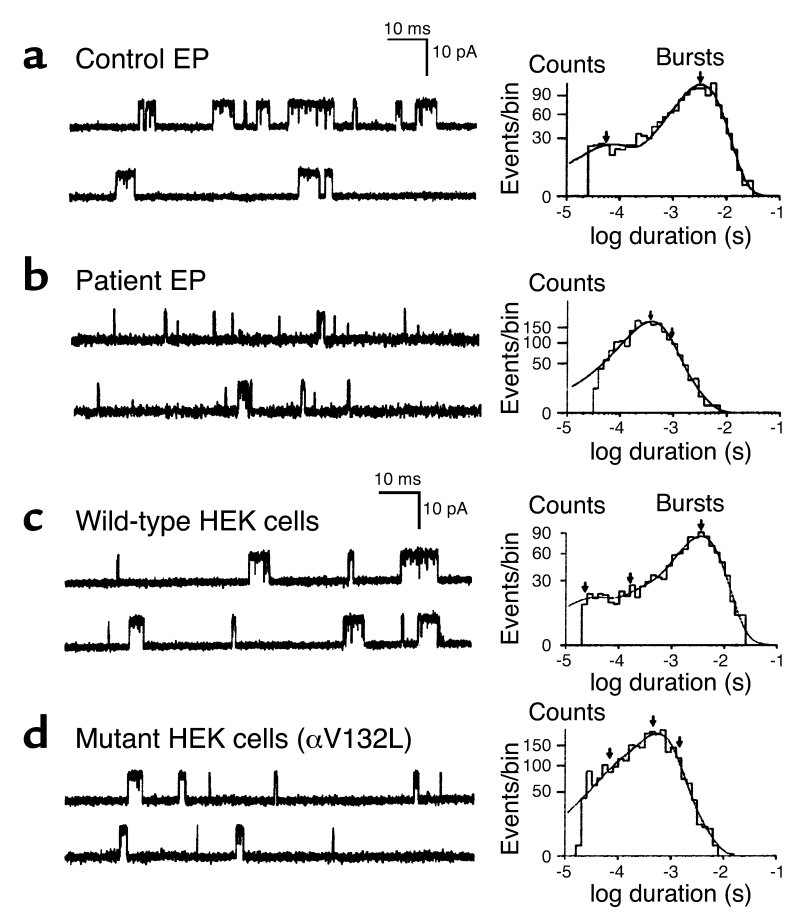

Figure 2

Channel events elicited by $50 \mathrm{nM}$ ACh from control (a) and patient EPs (b), and from HEK cells expressing wild-type (c) and mutant AChR's (d). Channel openings are upward deflections. Logarithmically binned burst duration histograms are fitted to the sum of exponentials. 


\section{a}

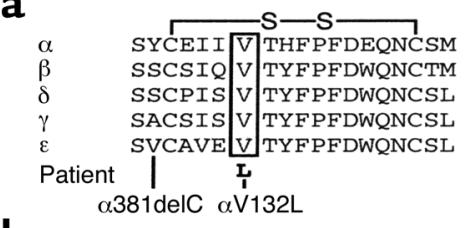

b

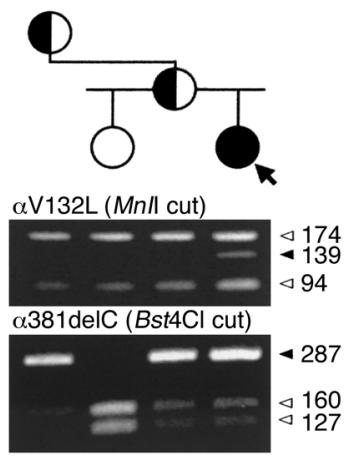

\section{Figure 3}

(a) Multiple alignment of cys-loop and its flanking regions. Valine 132 is conserved in all human AChR subunits and in $\alpha$ subunits of all species. (b) $\mathrm{Mnll}$ and $B s t 4 \mathrm{Cl}$ restriction analysis of PCR products amplified from genomic DNA of patient's and relatives' blood. Closed and open arrows point, respectively, to mutant and wild-type fragments. Unaffected grandmother and mother are heterozygous for $\alpha 381 \mathrm{del} C$, and a half sister carries no mutation. Father was not available for genetic analysis. Arrow and closed circle indicate patient; half-shaded circles represent asymptomatic carriers.

hold her head erect, stand, or walk. She has marked eyelid ptosis and facial diplegia, is unable to close her mouth, and cannot speak or swallow (Figure 1a). She has a $25-50 \%$ decrement of the fifth compared with the first evoked compound muscle fiber action potential, has no anti-AChR antibodies, and responds partially to anticholinesterase drugs and 3,4-diaminopyridine.

EP studies. EP configuration was normal, as judged from the cytochemical reaction product for AChE on longitudinally oriented teased single muscle fibers. Two-color fluorescence localization of AChR and $\mathrm{AChE}$ at the EPs revealed no abnormality. On electron microscopy, the structural integrity of the nerve terminals and junctional folds was preserved. Ultrastructural localization of AChR with peroxidaselabeled $\alpha$-bgt revealed normal density and distribution of AChR on the crests of the junctional folds (Figure 1b). The AChR index, defined as the ratio of the length of the postsynaptic membrane reacting for AChR to the length of the primary synaptic cleft (21), was normal (Table 1). The number of EP specific $\alpha$-bgt binding sites was close to the mean adult control value (Table 1).

Although AChR was normally abundant at the EPs by multiple criteria, the MEPP and MEPC amplitudes were so low that most were lost in baseline noise. Normally, $d$-tubocurarine is required to prevent twitching during recording of EPPs, but in the patient these could be recorded in its absence. The quantal content of the EPP $(m)$ fell in the range of values observed in adult controls. The MEPP and MEPC amplitudes, calculated from the respective quantal component of the EPP and EPC, were reduced to about $10 \%$ of normal (Table 1 ).

Single-channel patch-clamp recordings from EPs revealed AChR channels opening to a normal conductance of approximately 59 picosiemens. Analysis of bursts of channel openings elicited by low concentrations of ACh yielded dwell-time histograms that were best fitted by two exponential components: a major component whose duration was only $16 \%$ that of wild-type, and a longer minor component (Table 1 and Figure 2, $a$ and $b$ ). Thus the burst open durations predict an abnormally fast decay of the response to quantal release of ACh. The overall EP studies revealed no AChR deficiency, abnormally brief channel activation episodes, and a markedly decreased synaptic response to ACh.

Mutation analysis. To look for a genetic basis for the observed kinetic abnormality, we directly sequenced the AChR $\varepsilon$ and $\alpha$ subunits and detected two heterozygous mutations in the $\alpha$ subunit. The first mutation, $\alpha \mathrm{V} 132 \mathrm{~L}$, is a G-to-C transversion of nucleotide 394 that converts a valine to a leucine at codon 132 in the cysloop of the $\alpha$ subunit. Valine at codon 132 is conserved in all human AChR subunits (Figure 3a), and in AChR $\alpha$ subunits of all species. The second mutation is a $\mathrm{C}$ nucleotide deletion at position 381 ( $\alpha 381 \mathrm{delC})$ in the extracellular domain of the AChR $\alpha$ subunit that predicts 16 missense codons followed by a stop codon. Restriction analysis indicates that the mutations are recessive and heteroallelic (Figure $3 b$ ). The $\alpha$ V132L mutation was not detected in 200 normal alleles.

Expression studies in HEK cells. The total binding of $\left[{ }^{125} \mathrm{I}\right] \alpha$-bgt to mutant receptors on the cell surface was normalized to that measured for wild-type AChR. There was robust expression of $\alpha \mathrm{V} 132 \mathrm{~L}-\mathrm{AChR}$ compared with wild type (mean \pm SD: $135 \% \pm 12 \%, n=3$ ), but essentially no expression of $\alpha 381$ delC-AChR $(0.21 \% \pm 0.22 \%, n=3$; $P<0.0001$ vs. wild type). Robust expression of $\alpha$ V132LAChR indicates that all three non- $\alpha$ subunits incorporate into surface receptors because omission of one or more subunits would abolish or markedly reduce expression $(35,36)$. Thus $\alpha 381 \mathrm{delC}$ is a null mutation and $\alpha \mathrm{V} 132 \mathrm{~L}$ determines the phenotype.

\section{Table 2}

Bursts of wild-type AChR and $\alpha \mathrm{V} 132 \mathrm{~L}-\mathrm{AChR}$ expressed in HEK cells

\begin{tabular}{lccc}
\hline & $\tau_{1}$ & $\tau_{2}$ & $\tau_{3}$ \\
Wild type & $0.036 \pm 0.0017^{\mathrm{A}}$ & $0.47 \pm 0.059$ & $\mathbf{3 . 3 1} \pm \mathbf{0 . 1 2}$ \\
$n=21$ & $(0.24 \pm 0.021)$ & $(0.21 \pm 0.027)$ & $(0.58 \pm 0.038)$ \\
$\alpha \mathrm{V} 132 \mathrm{~L}-A C h R$ & $0.080 \pm 0.0058$ & $\mathbf{0 . 5 0} \pm \mathbf{0 . 0 2 4}$ & $1.18 \pm 0.084$ \\
$n=7$ & $(0.15 \pm 0.019)$ & $(0.66 \pm 0.021)$ & $(0.19 \pm 0.019)$
\end{tabular}

Values indicate mean \pm SEM. $n$, number of patches. Values in parentheses represent fractional histogram areas. Values in bold indicate dominant burst component. ACh concentration was $50 \mathrm{nM}$, temperature was $22^{\circ} \mathrm{C} \pm 0.5^{\circ} \mathrm{C}$, and bandwidth was $11.7 \mathrm{kHz}$. ${ }^{A}$ Not detected at three patches. 


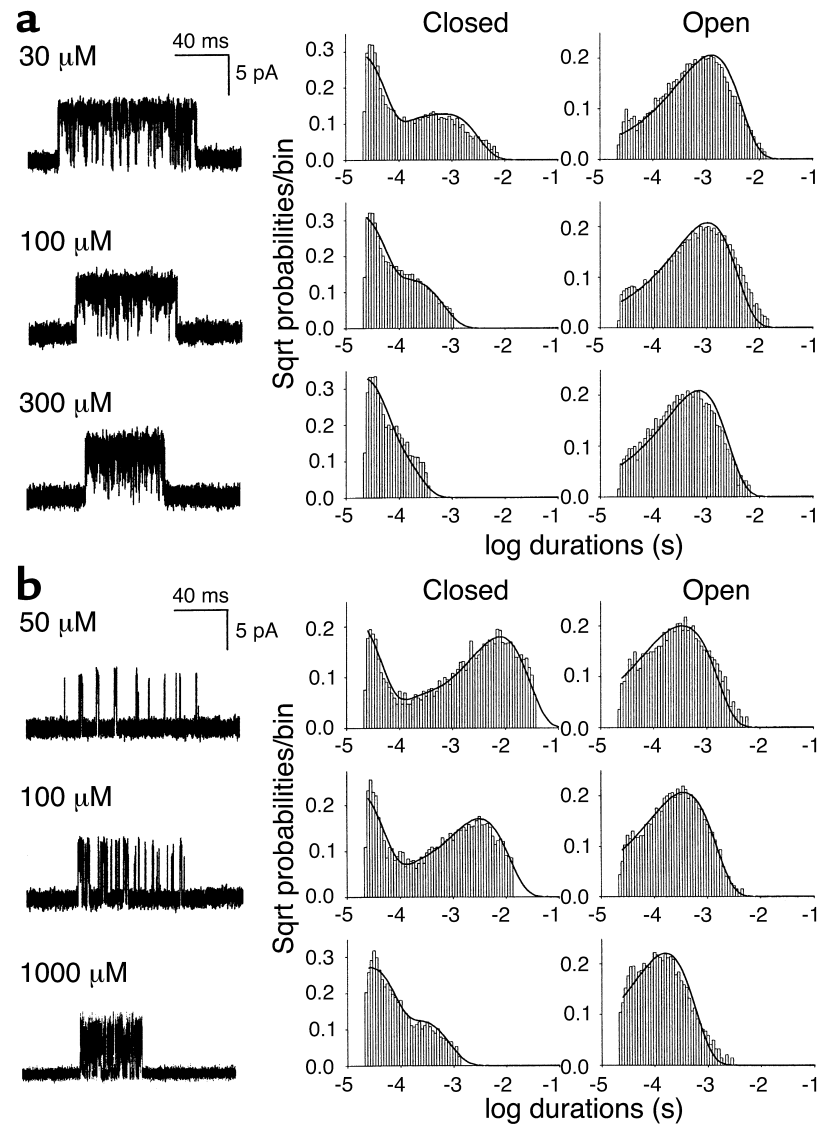

Figure 4

Kinetics of activation of wild-type (a) and $\alpha \mathrm{V} 132 \mathrm{~L}$ (b) AChR. Left column, individual clusters of single-channel currents recorded at indicated ACh concentrations from HEK cells. Currents are shown at a bandwidth of $11.7 \mathrm{kHz}$, with channel openings as upward deflections. Center and right columns show corresponding histograms of closed and open durations with superimposed global fits for Scheme 1 (see Figure 5) for the entire range of ACh concentrations. Fitted rate constants are given in Table 3 . Sqrt, square root.

To compare the affinity of ACh for $\alpha \mathrm{V} 132 \mathrm{~L}$ and wild-type receptors, we measured ACh binding at steady state by competition against the initial rate of [ $\left.{ }^{125} \mathrm{I}\right] \alpha$-bgt binding to intact cells. The apparent dissociation constant of $\alpha$ V132L-AChR $\left(6.18 \times 10^{-7}\right)$ increased by fivefold relative to wild-type $\left(1.26 \times 10^{-7}\right)$, but the Hill coefficients were similar (1.2 and 1.4, respectively). The increased dissociation constant suggests that $\alpha \mathrm{V} 132 \mathrm{~L}$ alters $\mathrm{ACh}$ binding, channel gating, desensitization, or a combination of these $(4,5)$.

Kinetics of activation of the $\alpha V 132 \mathrm{~L}$ mutant. To determine whether $\alpha \mathrm{V} 132 \mathrm{~L}$ causes the fast-channel CMS, we expressed the $\alpha \mathrm{V} 132 \mathrm{~L}$ mutant with complementary wild-type subunits in HEK 293 cells, recorded single-channel currents activated by a low concentration of ACh $(50 \mathrm{nM})$, and compared these with singlechannel currents recorded from control and patient EPs (Figure 2). As observed at patient EPs, singlechannel currents through the engineered mutant AChR activate in bursts of much shorter duration
(Figure 2, c and d). Analysis of the dwell-time histograms revealed three components of channel opening bursts for both wild-type and $\alpha$ V132L-AChR's. The dominant component of the $\alpha \mathrm{V} 132 \mathrm{~L}$ bursts was some sevenfold briefer than that of wild-type bursts (Table 2 and Figure 2, c and d). Moreover, the mean duration of this component in the $\alpha \mathrm{V} 132 \mathrm{~L}$ mutant $(0.50 \mathrm{~ms})$ was essentially identical to that obtained from patient EPs (0.44 ms; see Table 1).

To identify kinetic steps in receptor activation altered by $\alpha \mathrm{V} 132 \mathrm{~L}$, we analyzed open and closed dwell times within clusters of channel openings elicited by a range of desensitizing concentrations of ACh (see Methods). Well-defined clusters of events were observed for wild-type AChR at concentrations of ACh greater than $10 \mu \mathrm{M}$, but the threshold for forming clusters for the $\alpha \mathrm{V} 132 \mathrm{~L}-\mathrm{AChR}$ was $50 \mu \mathrm{M} \mathrm{ACh}$, indicating resistance to desensitization (Figure 4, left column), as previously described for the fast-channel mutation $\varepsilon P 121 \mathrm{~L}$ (4). For both wild-type and mutant receptors, the dominant component of closed dwell times shifts to shorter durations as the ACh concentration increases, but at all $\mathrm{ACh}$ concentrations this component is prolonged in the mutant compared with the wild-type (Figure 4, center column). Open-time histograms were independent of ACh concentration, but open dwell times for the $\alpha \mathrm{V} 132 \mathrm{~L}$ mutant were shifted to briefer durations compared with wild type (Figure 4, right column). Thus the $\alpha \mathrm{V} 132 \mathrm{~L}$ mutation reduces the probability of channel opening over the range of ACh concentrations examined.

We next analyzed the open and closed dwell times according to a linear scheme of receptor activation that recognizes a monoliganded and diliganded open state of the receptor (Scheme 1; see Figure 5).

In this scheme, two agonists (A) bind to the receptor (R) with association rate constants $k_{+1}$ and $k_{+2}$, and dissociate with rate constants $k_{-1}$ and $k_{-2}$. Receptors occupied by one agonist open with rate $\beta_{1}$ and close with rate $\alpha_{1}$, while receptors occupied by two agonists open with rate $\beta_{2}$ and close with rate $\alpha_{2}$. Asterisks indicate open state of the receptor. $\mathrm{R}_{\mathrm{B}}$ indicates blocked state of the receptor. At high concentrations, ACh blocks the open channel with rate $k_{+\mathrm{b}}$ and unblocks with rate $k_{-\mathrm{b}}$. The rate constants computed by maximum likelihood (see Methods) allow calculation of equilibrium dissociation constants $\left(K_{\mathrm{n}}=k_{-\mathrm{n}} / k_{+\mathrm{n}}\right)$ and channel gating equilibrium constants $\left(\theta_{n}=\beta_{n} / \alpha_{n}\right)$.

Kinetic analysis of currents through the $\alpha \mathrm{V} 132 \mathrm{~L}$ receptor yielded a complete set of rate constants describing the activation process (Table 3). Probability

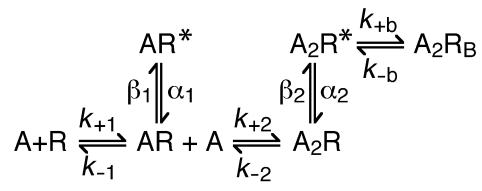

Figure 5

Scheme 1 of AChR activation. 
Table 3

Activation kinetics of wild-type and mutant AChR's expressed in HEK cells

\begin{tabular}{|c|c|c|c|c|c|c|c|c|c|c|c|c|c|c|}
\hline & $k_{+1}$ & $k_{-1}$ & $K_{1} / \mu \mathrm{M}$ & $k_{+2}$ & $k_{-2}$ & $K_{2} / \mu \mathrm{M}$ & $\beta_{1}$ & $\alpha_{1}$ & $\theta_{1}$ & $\beta_{2}$ & $\alpha_{2}$ & $\theta_{2}$ & $k_{+\mathrm{b}}$ & $k_{\text {-b }} \quad K_{\mathrm{B}} / \mathrm{mM}$ \\
\hline Wild-type & $\begin{array}{c}102 \pm \\
8\end{array}$ & $\begin{array}{c}1,951 \pm \\
200\end{array}$ & 19 & $\begin{array}{c}88 \pm \\
2\end{array}$ & $\begin{array}{c}10,363 \pm \\
169\end{array}$ & 117 & $\begin{array}{c}196 \pm \\
15\end{array}$ & $\begin{array}{c}3,066 \pm \\
250\end{array}$ & .064 & $\begin{array}{c}54,240 \pm \\
1,094\end{array}$ & $\begin{array}{c}2,136 \pm \\
52\end{array}$ & 25 & $\begin{array}{c}133.96 \pm \\
5.26\end{array}$ & $\begin{array}{c}171,415 \pm 1.28 \\
1,819\end{array}$ \\
\hline$\alpha \mathrm{V} 132 \mathrm{~L}$ & $\begin{array}{c}37 \pm \\
6\end{array}$ & $\begin{array}{l}2,712 \pm \\
485\end{array}$ & 73 & $\begin{array}{c}8 \pm \\
0.36\end{array}$ & $\begin{array}{c}31,269 \pm \\
894\end{array}$ & 3,909 & $\begin{array}{c}92 \pm \\
8\end{array}$ & $\begin{array}{c}7,869 \pm \\
397\end{array}$ & 0.012 & $\begin{array}{c}54,911 \pm \\
4,190\end{array}$ & $\begin{array}{c}4,587 \pm \\
277\end{array}$ & 12 & $\begin{array}{c}14.40 \pm \\
0.72\end{array}$ & $\begin{array}{l}53,214 \pm 3.70 \\
2,816\end{array}$ \\
\hline$\alpha \mathrm{V} 132 \mathrm{I}$ & $\begin{array}{c}39 \pm \\
5\end{array}$ & $\begin{array}{c}643 \pm \\
92\end{array}$ & 16 & $\begin{array}{l}16 \pm \\
0.50\end{array}$ & $\begin{array}{c}26,668 \pm \\
518\end{array}$ & 1,667 & $\begin{array}{c}45 \pm \\
3\end{array}$ & $\begin{array}{c}4,925 \pm \\
257\end{array}$ & 0.0091 & $\begin{array}{c}30,044 \pm \\
2,095\end{array}$ & $\begin{array}{c}2,554 \pm \\
85\end{array}$ & 12 & $\begin{array}{c}34.07 \pm \\
9.35\end{array}$ & $\begin{array}{c}107,063 \pm 3.14 \\
1,628\end{array}$ \\
\hline$\delta \mathrm{V} 134 \mathrm{~L}$ & $\begin{array}{c}174 \pm \\
6\end{array}$ & $\begin{array}{l}8,323 \pm \\
548\end{array}$ & 48 & $\begin{array}{c}180 \pm \\
8\end{array}$ & $\begin{array}{c}18,475 \pm \\
382\end{array}$ & 103 & $\begin{array}{c}1,417 \pm \\
99\end{array}$ & $\begin{array}{c}=9,403 \pm \\
268\end{array}$ & 0.15 & $\begin{array}{c}33,083 \pm \\
1,133\end{array}$ & $\begin{array}{c}4,489 \pm \\
106\end{array}$ & 7 & $\begin{array}{c}181.78 \pm \\
6.61\end{array}$ & $\begin{array}{c}165,034 \pm 0.91 \\
1,908\end{array}$ \\
\hline$\beta \vee 132 L$ & $\begin{array}{c}84 \pm \\
7\end{array}$ & $\begin{array}{c}1,143 \pm \\
137\end{array}$ & 13 & $\begin{array}{c}68 \pm \\
2\end{array}$ & $\begin{array}{c}8,245 \pm \\
149\end{array}$ & 121 & $\begin{array}{c}76 \pm \\
12\end{array}$ & $\begin{array}{c}1,526 \pm \\
200\end{array}$ & 0.050 & $\begin{array}{c}46,735 \pm \\
1,542\end{array}$ & $\begin{array}{c}1,542 \pm \\
35\end{array}$ & 30 & $\begin{array}{c}104.77 \pm \\
5.04\end{array}$ & $\begin{array}{c}=135,010 \pm 1.29 \\
2,143\end{array}$ \\
\hline EV132L & $\begin{array}{c}90 \pm \\
5\end{array}$ & $\begin{array}{c}2,013 \pm \\
185\end{array}$ & 22 & $\begin{array}{c}116 \pm \\
3\end{array}$ & $\begin{array}{c}10,454 \pm \\
207\end{array}$ & 90 & $\begin{array}{c}417 \pm \\
35\end{array}$ & $\begin{array}{c}2,176 \pm \\
195\end{array}$ & 0.19 & $\begin{array}{c}44,334 \pm \\
948\end{array}$ & $\begin{array}{c}1,596 \pm \\
37\end{array}$ & 28 & $\begin{array}{c}56.30 \pm \\
3.24\end{array}$ & $\begin{array}{c}114,968 \pm 2.04 \\
2,467\end{array}$ \\
\hline
\end{tabular}

Association rate constants are in $\mu \mathrm{M}^{-1} \mathrm{~s}^{-1}$, and all other rate constants are in s-1. The channel gating equilibrium constants $\theta_{1}$ and $\theta_{2}$ are the ratios of the opening to closing rate constants, $\beta_{1} / \alpha_{1}$ and $\beta_{2} / \alpha_{2}$, respectively.

density functions computed from the fitted rate constants superimpose upon open and closed time histograms obtained for each ACh concentration, showing that activation of both mutant and wild-type receptors is well described by Scheme 1 (see Figure 5). The predominant effect of $\alpha \mathrm{V} 132 \mathrm{~L}$ is a reduced affinity of ACh for the resting, closed state of the receptor. The association rate constants $k_{+1}$ and $k_{+2}$ are slowed, and the dissociation rate constants $k_{-1}$ and $k_{-2}$ are increased, yielding increases in $K_{1}$ and $K_{2}$ of about fourfold and 30-fold, respectively. Unexpectedly, although the $\alpha \mathrm{V} 132 \mathrm{~L}$ mutation is present in both $\alpha$ subunits of the mutant AChR, the second binding step is affected much more than the first. Gating efficiency of the doubly occupied receptor is reduced to $48 \%$ of the wild-type value.

The rate constants in Table 3 allow calculation of several functional correlates of the active state of the receptor. Neglecting the blocked state, the mean duration of a single opening of the diliganded receptor is $1 / \alpha_{2}$; the number of openings per burst is $\left(1+\beta_{2} / k_{-2}\right)$; and the mean burst duration is $\left(\beta_{2} / k_{-2}+1\right) / \alpha_{2}(37)$. Given these relationships, comparison of wild-type versus $\alpha \mathrm{V} 132 \mathrm{~L}$ AChR's predicts $0.47 \mathrm{~ms}$ versus 0.22 $\mathrm{ms}$ for the duration of a diliganded opening, 6.0 versus 2.8 openings per burst, and $2.83 \mathrm{~ms}$ versus 0.60 $\mathrm{ms}$ for the burst duration. The burst duration predicted from kinetic analysis is similar to that observed at the EPs (wild type, $3.04 \mathrm{~ms}$; CMS patient, $0.44 \mathrm{~ms}$ ) and to that observed in transfected HEK cells at limiting low concentrations of ACh (wild-type, $3.31 \mathrm{~ms}$; mutant, $0.50 \mathrm{~ms}$ ).

Comparison of $\alpha V 132 L$ and $\alpha V 132 I$. We previously reported mutations of $\alpha \mathrm{V} 285$ in the third transmembrane domain that showed stereochemical contributions to channel gating. We found that gating efficiency decreased linearly with increasing volume of the moiety attached to the $\beta$ carbon of the side chain of the substituting residue (7). Thus, leucine with an isopropyl group on its $\beta$ carbon hindered gating more severely than did isoleucine with a smaller ethyl group on its $\beta$ carbon (7). To determine whether such a stereochemical contribution underlies the functional consequences of $\alpha \mathrm{V} 132$, we compared the kinetics of the $\alpha \mathrm{V} 132 \mathrm{~L}$ and $\alpha \mathrm{V} 132 \mathrm{I}$ mutants. Analysis of open and closed dwell times for the $\alpha$ V132I-AChR yielded well-defined rate constants for Scheme 1. The isoleucine mutation reduces gating efficiency to the same extent as the leucine mutation but affects $\mathrm{ACh}$ binding to the resting closed state less than does the leucine mutation; $K_{2}$ decreases by only about 14 -fold and $K_{1}$ is unaltered (Table 3 ). Thus, although overall side-chain volume is equivalent for leucine and isoleucine, the smaller ethyl substitution on the $\beta$ carbon of isoleucine may explain the smaller effect on ACh binding. Alternatively, the

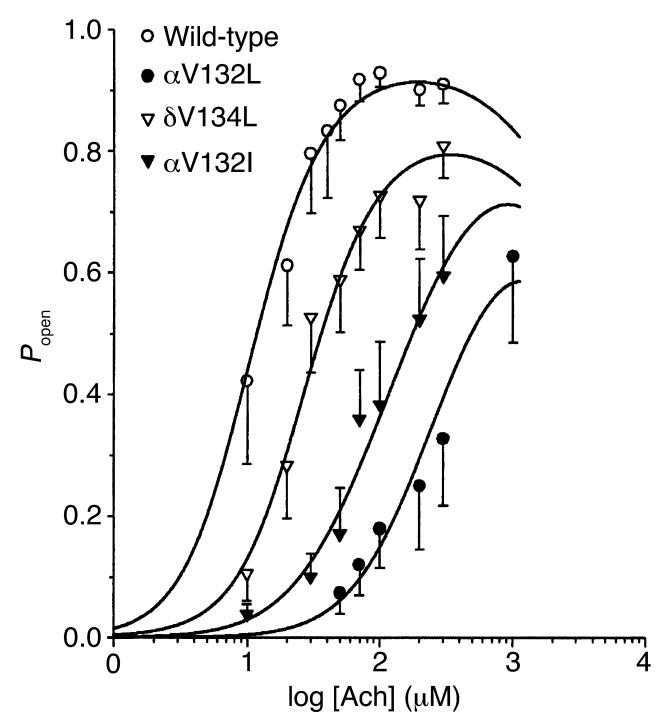

\section{Figure 6}

Open probability $\left(P_{\text {open }}\right)$ as a function of ACh concentration. Symbols represent mean $P_{\text {open }}$ computed for 75-311 clusters in a single patch. Error bars indicate standard deviation. Smooth curves are the predicted relationships computed from the rate constants in Table 3. Open circles, wild type; filled circles, $\alpha \mathrm{V} 132 \mathrm{~L}$ mutant; open inverted triangles, $\delta \mathrm{V} 134 \mathrm{~L}$ mutant; filled inverted triangles, $\alpha \mathrm{V} 132 \mathrm{I}$ mutant. 


$$
\mathrm{AR}+\mathrm{A} \underset{k_{-2}}{\stackrel{k_{+2}}{\rightleftharpoons}} \mathrm{A}_{2} \mathrm{R} \stackrel{\beta_{2}}{\longrightarrow} \mathrm{A}_{2} \mathrm{R}^{*}
$$

Figure 7

Scheme 2 of AChR activation.

different branching patterns of the leucine and isoleucine side chains may be responsible for the different functional consequences.

Mutations of equivalent residues in non- $\alpha$ subunits. To determine whether cys-loops of the non- $\alpha$ subunits contribute similarly to the kinetics of receptor activation, we substituted leucine for valine at positions equivalent to $\alpha 132$ in AChR non- $\alpha$ subunits and analyzed single-channel dwell times from the resulting mutant receptors. Single-channel dwell times from the $\beta V 132 \mathrm{~L}, \varepsilon V 132 \mathrm{~L}$, and $\delta \mathrm{V} 134 \mathrm{~L}$ mutants also yielded well-defined rate constants in Scheme 1. The equivalent mutation in the $\delta$ subunit, $\delta \mathrm{V} 134 \mathrm{~L}$, has only a minor effect on ACh affinity, but it impairs gating efficiency of the doubly liganded receptor: $\theta_{2}$ is reduced to $28 \%$ of the wild-type counterpart, and the predicted burst duration falls from about $3 \mathrm{~ms}$ for wild type to $0.62 \mathrm{~ms}$ for the mutant. By contrast to mutations in $\alpha$ and $\delta$ subunits, the corresponding valine to leucine mutations in the $\beta$ and $\varepsilon$ subunits do not affect the kinetics of receptor activation (Table 3). Thus, the overall findings demonstrate functional asymmetry in the contributions of cysloops from different AChR subunits.

Effects of the cys-loop mutants on channel open probability. Next we compared fractional activation of wildtype and mutant receptors as a function of ACh concentration. Fractional activation is taken as the probability that a channel is open $\left(P_{\text {open }}\right)$ within a defined cluster of channel events; for each concentration of ACh, the mean $P_{\text {open }}$ computed for all clusters is plotted (Figure 6). Each of the mutations, $\alpha$ V132L, $\alpha$ V132I, and $\delta$ V134L, shifts the $P_{\text {open }}$ curve to higher concentrations of ACh, with $\alpha \mathrm{V} 132 \mathrm{~L}$ identified as having the most severe effect. Corresponding $P_{\text {open }}$ curves for the $\beta V 132 \mathrm{~L}$ and $\varepsilon V 132 \mathrm{~L}$ mutations did not differ significantly from wild type (data not shown). Theoretical $P_{\text {open }}$ curves computed from the fitted rate constants superimpose upon the $P_{\text {open }}$ measurements, further supporting the validity of the estimated rate constants in Table 3.

\section{Discussion}

We trace the clinical phenotype of a highly disabling fast-channel CMS to two heteroallelic mutations in the AChR $\alpha$ subunit: a missense mutation, $\alpha \mathrm{V} 132 \mathrm{~L}$, in the signature cys-loop, and a frame-shifting null mutation, 381delC, that unmasks the functional consequences of $\alpha \mathrm{V} 132 \mathrm{~L}$. The robust expression of the $\alpha \mathrm{V} 132 \mathrm{~L}-\mathrm{AChR}$ at EPs and in HEK cells indicates that the pathologic consequences stem entirely from abnormal activation kinetics of the mutant channels.
Neuromuscular transmission is compromised by the very small and rapidly decaying synaptic currents resulting from abnormally brief single-channel activation episodes that occur with low probability. Singlechannel kinetic analysis reveals that, compared with wild-type, $\alpha \mathrm{V} 132 \mathrm{~L}$ impairs ACh binding to receptors in the resting closed state by about 30 -fold, but attenuates channel gating by only about twofold. Mutation of the equivalent valine residue in the $\delta$ subunit has little effect on ACh binding but reduces channel gating efficiency about 3.6-fold, while corresponding mutations in the $\beta$ and $\varepsilon$ subunits are without effect. The overall findings reveal functional asymmetry between cysloops of the different AChR subunits in contributing to ACh binding and channel gating.

We can assess the consequences of the mutation on neuromuscular transmission using our estimated receptor activation rate constants (Table 3). Both the amplitude and the decay rate of the EPC determine the magnitude of the postsynaptic response. The usual approximation to assess changes in EPC amplitude is the probability that a receptor will open following full occupancy of the binding sites by the impulse of ACh, which is given by $\beta_{2} /\left(\beta_{2}+k_{-2}\right)$. However, for the mutant receptor, the assumption of full occupancy is likely not valid because the rate of ACh association is slowed by an order of magnitude at the second occupancy step of Scheme 1 . We therefore used our estimated rate constants to compute the fraction of active receptors following an impulse of ACh according to a subset of Scheme 1 (Scheme 2; see Figure 7).

Scheme 2 neglects the return transition from the open state because it is too slow to contribute to the peak of the MEPC. We also make the further assumption that the first ACh binding step, which is only slightly altered in the mutant, is complete at the instant of the pulse of ACh. Given these simplifying assumptions, the fraction of active receptors as a
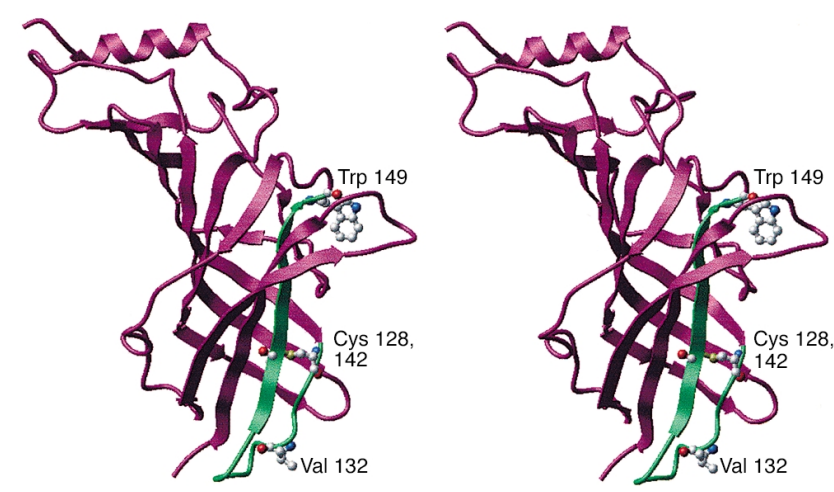

Figure 8

Location of $\mathrm{V} 132$ in the $\mathrm{AChR} \alpha$ subunit. Stereo view of a structural model of the human $\alpha$ subunit (42) is shown in magenta with the cysloop and contiguous $\beta$ strand 7 highlighted in green. Ball and stick representations indicate valine (Val) 132 within the cys-loop, cysteines (Cys) 128 and 142 which form the loop, and tryptophan (Trp) 149 at the center of the $\alpha$ subunit portion of the binding pocket. 
function of time can be predicted by writing equations for the time dependence of each receptor state and solving the set of equations using the Laplace transform to yield

\section{Equation 1}

$$
\begin{aligned}
{\left[A_{2} R^{*}\right](t)=} & 1-1 / 2 \lambda_{1}\left\{\left(\lambda_{2}+\lambda_{1}\right) \exp -\left\{(1 / 2)\left(\lambda_{2}-\lambda_{1}\right)(t)\right\}-\right. \\
& \left.\left(\lambda_{2}-\lambda_{1}\right) \exp -\left\{(1 / 2)\left(\lambda_{2}+\lambda_{1}\right)(t)\right\}\right\}
\end{aligned}
$$

where $\lambda_{1}=\operatorname{sqrt}\left\{\left(k_{+2}[\mathrm{ACh}]+k_{-2}+\beta_{2}\right)^{2}-4 k_{+2}[\mathrm{ACh}] \beta_{2}\right\}$ and $\lambda_{2}=\left(k_{+2}[\mathrm{ACh}]+k_{-2}+\beta_{2}\right)$.

Given that the synaptic ACh concentration is a square wave pulse with a concentration amplitude of $1 \mathrm{mM}$ and duration of $50-80 \mu \mathrm{s}(38,39)$, our fitted rate constants predict activation of $78 \%$ of wild-type receptors and $14 \%$ of mutant receptors following an impulse of ACh, corresponding to an $18 \%$ reduction of the MEPC amplitude relative to wild type. This predicted reduction of the MEPC approaches our observed reduction of $8 \%$, but the difference between predicted and experimental amplitudes would vanish with only a twofold reduction of the peak $\mathrm{ACh}$ concentration, the duration of the ACh transient, or a combination of these. Elementary functional steps responsible for this reduction of the MEPC amplitude are the tenfold reduced rate of ACh association in the second binding step of Scheme 1 and the threefold increased rate of ACh dissociation from the diliganded receptor.

Neuromuscular transmission is further compromised by the rapid decay of MEPCs caused by the mutation. The MEPC decay time constant is approximated by the mean duration of bursts of single-channel openings elicited by a low concentration of $\mathrm{ACh}$, and is given by $\left(1+\beta_{2} / k_{-2}\right) / \alpha_{2}$. In the mutant receptor, the threefold increase of $k_{-2}$ and twofold increase of $\alpha_{2}$ reduce mean burst duration from $2.8 \mathrm{~ms}$ for wild type to $0.6 \mathrm{~ms}$ for the $\alpha \mathrm{V} 132$ mutant. The shortened burst duration alone predicts reduction of the total current flow during an EPC to $21 \%$ of that of the wild-type receptor.

To summarize, we attribute the extremely severe phenotypic consequences of $\alpha \mathrm{V} 132 \mathrm{~L}$ to the approximately 30 -fold decrease of ACh binding affinity for the second of two closed-state binding sites, so that the prevailing ACh concentration in the synaptic space saturates only about $10 \%$ of these sites in the mutant receptor, and to a further decrease of about fivefold in the total ACh-induced current due to an accelerated decay of the synaptic response.

Our mechanistic findings can be interpreted in light of the crystal structure of an ACh binding protein from a fresh water mollusk (18), together with a structural model of the receptor counterpart based on ACh binding protein and lysine scanning mutagenesis (40). ACh binding protein is a homopentamer of $\alpha$-like subunits that are $24 \%$ homologous to the neuronal $\alpha_{7}$ subunit. Moreover, ACh binding protein contains many of the structural cornerstones that give nicotinic receptors their unique signature, including ligand-binding sites at interfaces between subunits, key aromatic residues at the ligand-binding sites, and the signature cys-loop formed between cysteines 128 and 142 of the muscle receptor $\alpha$ subunit. In the structural model of the human AChR ligand-binding domain, the cys-loop is located at the bottom of each subunit, bridging $\beta$ strands 6 and 7, and is directly connected to $\alpha \mathrm{W} 149$, the center of the binding site through $\beta$ strand 7 (40) (see Figure 8). Moreover, the cys-loop is located at the junction between ligand-binding and transmembrane domains (41), and recent cryoelectron microscopy studies suggest that it may be in close apposition with the second and third transmembrane domains (42), where it may couple structural changes at the binding site to opening and closing of the channel gate. Our observation that the cys-loop in the $\alpha$ subunit contributes primarily to ACh binding is explained by its location on the same $\beta$ strand that harbors $\alpha \mathrm{W} 149$ at the center of the binding site (Figure 8), which is the most likely residue to stabilize the quaternary ammonium group of ACh (43). Insertion of the larger leucine side chain in the cys-loop likely displaces the $\beta$ strand, and consequently $\alpha \mathrm{W} 149$, thus causing low affinity for ACh. Consistent with this notion is that the isoleucine mutation, which has a smaller ethyl rather than isopropyl substitution on its $\beta$ carbon, has a smaller effect on ACh binding.

Unexpectedly, the mutation in the $\alpha$ subunit cysloop reduces gating efficiency only about twofold, compared with an approximately 30 -fold effect on ACh binding. A strong gating effect might be expected because of the proximity of the cys-loop to the transmembrane domains. However, the equivalent mutation in the $\delta$ subunit cys-loop attenuates channel gating nearly fourfold, with little effect on ACh binding. The $\delta \mathrm{V} 134$ mutation and the contiguous $\beta$ strand 7 are far away from the ACh binding site, and would not be expected to affect ACh binding. The functional consequences of the mutation in the $\delta$ subunit cysloop suggest similar effects following mutation of equivalent residues in $\beta$ and $\varepsilon$ subunits, but these show no effect. Therefore, although the four types of AChR subunits have similar folded structures, our findings demonstrate an unexpected functional asymmetry of the cys-loops in the different subunits.

\section{Acknowledgments}

This work was supported by grants from the NIH to A.G. Engel (NS-6277) and to S.M. Sine (NS-31744) and by a Muscular Dystrophy Association grant to A.G. Engel. We thank Zeljko Bajzer for providing Equation 1.

\footnotetext{
1. Engel, A.G., Ohno, K., and Sine, S.M. 1999. Congenital myasthenic syndromes. In Myasthenia gravis and myasthenic disorders. A.G. Engel, editor. Oxford University Press. New York, New York, USA. 251-297.

2. Ohno, K., and Engel, A.G. 2002. Congenital myasthenic syndromes: genetic defects at the neuromuscular junction. Curr. Neurol. Neurosci. Rep. 2:78-88.

3. Ohno, K., et al. 2002. Rapsyn mutations in humans cause endplate
} 
acetylcholine receptor deficiency and myasthenic syndrome. Am.J. Hum. Genet. 70:875-885.

4. Ohno, K., et al. 1996. Congenital myasthenic syndrome caused by decreased agonist binding affinity due to a mutation in the acetylcholine receptor $\varepsilon$ subunit. Neuron. 17:157-170.

5. Sine, S.M., et al. 2002. Naturally occurring mutations at the acetylcholine receptor binding site independently alter ACh binding and channel gating. J. Gen. Physiol. 120:483-496.

6. Brownlow, S., et al. 2001. Acetylcholine receptor $\delta$ subunit mutations underlie a fast-channel myasthenic syndrome and arthrogryposis multiplex congenita. J. Clin. Invest. 108:125-130. doi:10.1172/JCI200112935.

7. Wang, H.-L., et al. 1999. Acetylcholine receptor M3 domain: stereochemical and volume contributions to channel gating. Nat. Neurosci. 2:226-233.

8. Milone, M., et al. 1998. Mode switching kinetics produced by a naturally occurring mutation in the cytoplasmic loop of the human acetylcholine receptor $\varepsilon$ subunit. Neuron. 20:575-588.

9. Wang, H.-L., et al. 2000. Fundamental gating mechanism of nicotinic receptor channel revealed by mutation causing a congenital myasthenic syndrome. J. Gen. Physiol. 116:449-460.

10. Le Novere, N., and Changeux, J.-P. 2001. LGICdb: the ligand-gated ion channel database. Nucleic Acids Res. 29:294-295.

11. Mishina, M., et al. 1985. Location of functional regions of acetylcholine receptor alpha-subunit by site directed mutagenesis. Nature. 313:364-369.

12. Fu, D.-X., and Sine, S.M. 1996. Asymmetric contribution of the conserved disulfide loop to subunit oligomerization and assembly of the nicotinic acetylcholine receptor. J. Biol. Chem. 271:31479-31484.

13. Green, W.N., and Wanamaker, C.P. 1997. The role of the cystine loop in acetylcholine receptor assembly. J. Biol. Chem. 272:20945-20953.

14. Blount, P., and Merlie, J.P. 1990. Mutational analysis of muscle nicotinic acetylcholine receptor subunit assembly. J. Cell. Biol. 111:2613-2622.

15. Sumikawa, K., and Gehle, V.M. 1992. Assembly of mutant subunits of the acetylcholine receptor lacking the conserved disulfide loop structure. J. Biol. Chem. 267:6286-6290.

16. Walcott, E.C., and Sumikawa, K. 1996. A conserved disulfide loop facilitates conformational maturation in the subunits of the acetylcholine receptor. Brain Res. Mol. Brain Res. 41:289-300.

17. Criado, M., Sarin, V., Fox, J.L., and Lindstrom, J. 1986. Evidence that the acetylcholine binding site is not formed by the sequence $\alpha 127-143$ of the acetylcholine receptor. Biochemistry. 25:2839-2846.

18. Brejc, K., et al. 2001. Crystal structure of ACh-binding protein reveals the ligand-binding domain of nicotinic receptors. Nature. 411:269-276.

19. Hutchinson, D.O., et al. 1993. Congenital endplate acetylcholinesterase deficiency. Brain. 116:633-653.

20. Engel, A.G. 1994. The muscle biopsy. In Myology. A.G. Engel and C. Franzini-Armstrong, editors. McGraw-Hill Inc. New York, New York, USA. 822-831.

21. Engel, A.G. 1994. Quantitative morphological studies of muscle. In Myology. A.G. Engel and C. Franzini-Armstrong, editors. McGraw-Hill Inc. New York, New York, USA. 1018-1045.

22. Engel, A.G., Lindstrom, J.M., Lambert, E.H., and Lennon, V.A. 1977. Ultrastructural localization of the acetylcholine receptor in myasthenia gravis and in its experimental autoimmune model. Neurology. 27:307-315.

23. Engel, A.G., Nagel, A., Walls, T.J., Harper, C.M., and Waisburg, H.A. 1993 Congenital myasthenic syndromes. I. Deficiency and short open-time of the acetylcholine receptor. Muscle Nerve. 16:1284-1292.

24. Uchitel, O., et al. 1993. Congenital myasthenic syndromes. II. A syndrome attributed to abnormal interaction of acetylcholine with its receptor. Muscle Nerve. 16:1293-1301.
25. Elmqvist, D., and Quastel, D.M.J. 1965. A quantitative study of end-plate potentials in isolated human muscle. J. Physiol. (Lond.) 178:505-529.

26. Milone, M., Hutchinson, D.O., and Engel, A.G. 1994. Patch-clamp analysis of the properties of acetylcholine receptor channels at the normal human end plate. Muscle Nerve. 17:1364-1369.

27. Ohno, K., et al. 1995. Congenital myasthenic syndrome caused by prolonged acetylcholine receptor channel openings due to a mutation in the M2 domain of the $\varepsilon$ subunit. Proc. Natl. Acad. Sci. USA. 92:758-762.

28. Schoepfer, R., Luther, M.A., and Lindstrom, J. 1988. The human medulloblastoma cell line TE671 expresses a muscle-like acetylcholine receptor. Cloning of the alpha-subunit cDNA. FEBS Lett. 226:235-240.

29. Luther, M.A., et al. 1989. A muscle acetylcholine receptor is expressed in the human cerebellar medulloblastoma cell line TE671. J. Neurosci. 9:1082-1096.

30. Ohno, K., et al. 1997. Congenital myasthenic syndromes due to heteroallelic nonsense/missense mutations in the acetylcholine receptor $\varepsilon$ subunit gene: identification and functional characterization of six new mutations. Hum. Mol. Genet. 6:753-766.

31. Bouzat, C., Bren, N., and Sine, S.M. 1994. Structural basis of different gating kinetics of fetal and adult acetylcholine receptors. Neuron. 13:1395-1402.

32. Sakmann, B., Patlak, J., and Neher, E. 1980. Single acetylcholine-activated channels show burst kinetics in the presence of desensitizing concentrations of agonist. Nature. 286:71-73.

33. Qin, F., Auerbach, A., and Sachs, F. 1996. Estimating single-channel kinetic parameters from idealized patch-clamp data containing missed events. Biophys. J. 70:264-280.

34. Wang, H.-L., et al. 1997. Mutation in the M1 domain of the acetylcholine receptor alpha subunit decreases the rate of agonist dissociation. J. Gen. Physiol. 109:757-766.

35. Mishina, M., et al. 1984. Expression of functional acetylcholine receptor from cloned cDNAs. Nature. 307:604-608.

36. Quiram, P., et al. 1999. Mutation causing congenital myasthenia reveals acetylcholine receptor $\beta / \delta$ subunit interaction essential for assembly. J. Clin. Invest. 104:1403-1410.

37. Colquhoun, D., and Hawkes, A.G. 1995. The principles of the stochastic interpretation of ion channel mechanisms. In Single-channel recording. B. Sakmann and E. Neher, editors. Plenum Press. New York, New York, USA. 397-482.

38. Stiles, J.R., Kovyazina, I.V., Salpeter, E.E., and Salpeter, M.M. 1999. The temperature sensitivity of miniature endplate currents is mostly governed by channel gating: evidence from optimized recordings and Monte Carlo simulations. Biophys. J. 77:1177-1187

39. Stiles, J.R., Van Helden, D., Bartol, T.M., Salpeter, E.E., and Salpeter, M.M. 1996. Miniature endplate current rise times $<100 \mu$ s from improved dual recordings can be modeled with passive acetylcholine diffusion from a synaptic vesicle. Proc. Natl. Acad. Sci. USA. 93:5747-5752.

40. Sine, S.M., Wang, H.-L., and Bren, N. 2002. Lysine scanning mutagenesis delineates structure of nicotinic receptor binding domain. J. Biol. Chem. 277:29210-29223.

41. Dougherty, D.A., and Lester, H.A. 2001. Snails, synapses and smokers. Nature. 411:252-253.

42. Unwin, N., Miyazawa, A., Li, J., and Fujiyoshy, Y. 2002. Activation of the nicotinic acetylcholine receptor involves a switch in conformation of the $\alpha$ subunits. J. Mol. Biol. 319:1165-1176.

43. Zhong, W., et al. 1998. From ab initio quantum mechanics to molecular neurobiology: a cation- $\pi$ binding site in the nicotinic receptor. Proc. Natl. Acad. Sci. USA. 95:12088-12093. 